\title{
DFT+DMFT study of spin-charge-lattice coupling in covalent $\mathrm{LaCoO}_{3}$
}

\author{
Hyowon Park ${ }^{1,2}$, Ravindra Nanguneri ${ }^{1,3}$, Anh T. Ngo ${ }^{2}$ \\ ${ }^{1}$ Department of Physics, University of Illinois at Chicago, Chicago, IL 60607, USA \\ ${ }^{2}$ Materials Science Division, Argonne National Laboratory, Argonne, IL, 60439, USA \\ ${ }^{3}$ Department of Chemistry, Brown University, Providence, RI 02912, USA
}

(Dated: May 12, 2020)

\begin{abstract}
We study energetics and the nature of both homogeneous and mixed spin (MS) states in $\mathrm{LaCoO}_{3}$ incorporating structural changes of the crystal volume expansion and the Co-O bond disproportionation (BD) during the spin-state transition using the density functional theory plus dynamical mean field theory (DFT+DMFT) method. DFT+DMFT predicts that energetics of both excited spin states are almost the same while DFT $+U$ calculations of the same structures energetically favor the MS states and produce various metastable solutions whose energetics depend sensitively on final spin states. Within DFT+DMFT, the homogeneous spin state in the expanded crystal volume shows the multiconfigurational nature with non-negligible occupancy probabilities of both high spin (HS) and low spin (LS) states along with $d^{6}$ and $d^{7}$ charge configurations indicating the dynamically fluctuating nature of spin and charge states due to the Co-O covalency. The nature of the MS state under the BD structure reveals that Co sites with the long Co-O bonds develop a Mott insulating state and favor HS with a $d^{6}$ configuration, while more covalent Co sites with the short Co-O bonds occupy more LS states with a $d^{7}$ configuration and behave as a band insulator, as a result, charge ordering is induced in the BD structure from the spin-state ordering. We also find that both energetics and electronic structure sensitively depend on the Co-O covalency effect, which can be tuned by changing the double counting potential and the resulting $d$-occupancy $\left(N_{d}\right)$, and $N_{d}$ close to 6.7 is consistent with the nature of the spin-state transition. Our results show that structural changes during the spin-state transition can play an important role in understanding energetics and electronic structure of $\mathrm{LaCoO}_{3}$.
\end{abstract}

\section{INTRODUCTION}

Transition metal oxides exhibit complex and rich phase diagrams arising from the strongly correlated nature of spin, charge, orbital, and lattice degrees of freedom ${ }^{1}$. $\mathrm{LaCoO}_{3}$ has been known for the spin-state transition of partially filled $d$ orbitals in a Co ion. At very low temperatures, $\mathrm{LaCoO}_{3}$ is a non-magnetic insulator with the low spin (LS) state. As the temperature is elevated above $90 \mathrm{~K}$, the magnetic susceptibility changes to a Curie-Weiss form indicating that paramagnetism dominates with higher spin states while retaining an insulating behavior ${ }^{2}$. The spin-state transition can be explained based on the atomic multiplet structure of the Co $d$ orbital, namely from the $\left|S_{z}\right|=0 \mathrm{LS}$ ground state to $\left|S_{z}\right|=1$ intermediate spin (IS) or $\left|S_{z}\right|=2$ high spin (HS) state. Various experimental results have been used to interpret the higher spin state as either $\mathrm{IS}^{3-6}$ or $\mathrm{HS}^{7}{ }^{-8}$. The MS of LS and HS has been also suggested to explain other experimental measurements ${ }^{9}-13$. Despite extensive experimental works, the nature of excited spin-states has not been clarified yet.

The spin-state transition in $\mathrm{LaCoO}_{3}$ occurs since the Hund's coupling tends to maximize the spin and excite electrons from $t_{2 g}$ to $e_{g}$ orbitals by overcoming the crystal field splitting between them. Here, the interplay between electron and lattice degrees of freedom plays an important role as the $e_{g}$ orbital occupation increases the $\mathrm{Co}-\mathrm{O}$ bond-length to reduce the repulsive Coulomb interaction of electrons between $\mathrm{Co}$ and $\mathrm{O}$ ions. This structural change also gives the positive feedback since the reduction of the crystal-field splitting can favor the spin- state transition. This strong electron-lattice coupling has been measured experimentally by the anomalous lattice expansion arising due to the $\mathrm{Co}-\mathrm{O}$ bond-length elongation at the spin-state transition ${ }^{14-17}$. Several scenarios of local structural distortions due to the spin excitation have been proposed although clear experimental evidences have not been given yet. The Co-O bonddisproportionation (BD) with alternating the long bond (LB) site and the short bond (SB) site $\frac{9,18}{18}$ was suggested to accommodate MS with HS and LS. The Jahn-Teller distortion 6,19 was also discussed possibly due to the IS state. The strong electron-lattice coupling has been also shown in the tensile-strained $\mathrm{LaCoO}_{3}$ film promoting various competing orders including $\operatorname{spin}^{20}-22$, $\operatorname{charge}^{23}$, and orbital orderings 24 .

Alongside experimental measurements, various theoretical scenarios based on first-principle calculations have been proposed to address this long-standing problem of the spin-state transition in $\mathrm{LaCoO}_{3}$. Density functional theory $(\mathrm{DFT})+\mathrm{U}$ calculations have been predicting that excited spin-states including $\operatorname{IS}^{25,26}$ and MS27,28 states can be energetically stable. Dynamical mean field theory (DMFT) can capture the multi-configurational nature of a paramagnetic state fluctuating dynamically beyond $\mathrm{DFT}+\mathrm{U}^{29,30}$. Early DFT+DMFT studies computed the single-particle spectra with spin-state crossover 31 comparable to the experimental X-ray absorption spectra 4 and also studied effects of the pressure 32 and the $\mathrm{Co}-\mathrm{O}$ covalency ${ }^{33}$ on the spin-state transition. Both the homogeneous spin excitation including the electronic entropy ${ }^{34}$ and the mixed LS and HS solution without any structural distortions 35,36 have been discussed as the possible origin 
of the spin-state transition within DFT+DMFT. However, no DFT+DMFT studies have addressed yet both the nature and energetics of paramagnetic states with all possible structural changes in this material.

In this paper, we adopt the DFT+DMFT method to study both homogeneous and MS states by incorporating structural changes during the spin-state transition. We show that the DFT + DMFT energetics treating the multi-configurational nature of a paramagnetic state can be noticeably different from the static DFT $+\mathrm{U}$ solutions in which various meta-stable spin-states are possible. We also find that both structural changes and the $\mathrm{Co}-\mathrm{O}$ covalency effect tuned by the double counting potential can strongly affect the electronic structure and energetics of spin-states in $\mathrm{LaCoO}_{3}$. In the expanded crystal volume, the occupation probabilities of higher spin-states in the Co ion increase as Co $d$ orbitals become more correlated. As temperature increases, DFT+DMFT can reproduce the insulator-to-metal transition, consistent with experiment, as the correlation in the Co ion becomes weaker. In the $\mathrm{BD}$ structure, the $\mathrm{Co}$ ion in the $\mathrm{Co}-\mathrm{O} \mathrm{LB}$ behaves as a Mott insulator with HS while the Co ion in the SB becomes a band insulator favoring LS. The charge ordering is also induced in the $\mathrm{BD}$ structure as the $\mathrm{LB}$ Co ion favors a $d^{6}$ configuration while the SB Co ion occupies more $d^{7}$ states. The charge-self-consistency in DFT+DMFT plays a role to reduce the charge ordering in the BD structure.

This paper is organized as follows. In Sec. II the method used in this paper is explained in details. Sec. IIIA discusses the energetics of possible spinstate transition in $\mathrm{LaCoO}_{3}$ comparing DFT+DMFT and $\mathrm{DFT}+\mathrm{U}$. The nature of the paramagnetic state treated in DFT+DMFT is shown in Sec.IIIB by computing the occupation probabilities of different spin-states. The origin of charge ordering driven by spin-state ordering in the $\mathrm{BD}$ structure is revealed in Sec $\amalg I C$ Results of the density of states computed using DFT and DFT+DMFT for different structures and temperatures are shown in Sec. IIID The self-energy data are displayed in Sec. IIIE and the effect of the charge-self-consistency in DFT+DMFT is also discussed in Sec.IIIF We summarize our paper with conclusions in Sec.IV]

\section{METHOD}

\section{A. Structural relaxation}

To study possible structural distortions during the spin-state transition, we first perform the structural relaxation by adopting the $\mathrm{DFT}+\mathrm{U}$ method as implemented in the Vienna ab-initio simulation package (VASP) code $\underline{37-40}^{\underline{40}}$ using different spin-states as initial guess. The Perdew-Burke-Ernzerhof (PBE) functional ${ }^{41}$ is used for the exchange-correlation functional within DFT. The DFT $+\mathrm{U}$ convergence is achieved using the plane-wave energy cut-off of $600 \mathrm{eV}$ and the $k$-point

\begin{tabular}{|c|c|c|c|c|c|}
\hline ures & S.G. & Vol. $\left[\tilde{A}^{3}\right]$ & $\mathrm{Co}-\mathrm{O}[\tilde{\mathrm{A}}]$ & Mom. $\left[\mu_{B}\right.$ & $N_{d}$ \\
\hline S1(LS) & $R 3 c$ & 56.40 & 1.95 & 0 & 7.1 \\
\hline S2(IS) & $R 3 c$ & 57.98 & 1.97 & 2.2 & 7.1 \\
\hline S3(MS) Co1 & $R 3$ & 57.98 & 1.99 & 3.0 & 6.9 \\
\hline S3(MS) Co2 & $R 3$ & 57.98 & 1.94 & 0.3 & 7.2 \\
\hline
\end{tabular}

TABLE I. The structural information of $\mathrm{LaCoO}_{3}$ relaxed structures obtained using $\mathrm{DFT}+\mathrm{U}$ with $\mathrm{U}=6 \mathrm{eV}$ and $\mathrm{J}=0.9 \mathrm{eV}$. Three different structures are obtained by relaxing with different magnetic moments and they are denoted as S1 (relaxed with LS), S2 (relaxed with IS), and S3 (relaxed with MS). The space group (S.G.), the crystal volume per formula unit, the Co-O bond length, magnetic moments, and the $d$-occupancy $\left(N_{d}\right)$ are given in this table.

mesh of $8 \times 8 \times 8$. The convergence of the structural relaxation is achieved if the atomic forces of all ions are smaller than $0.01 \mathrm{eV} / \AA$. Within $\mathrm{DFT}+\mathrm{U}$, we use the onsite Hubbard interaction $\mathrm{U}=6 \mathrm{eV}$, which is obtained using the constraint DFT method ${ }^{33}$, and the Hund's coupling $\mathrm{J}=0.9 \mathrm{eV}$. Although the ground-state of $\mathrm{LaCoO}_{3}$ is paramagnetic, $\mathrm{DFT}+\mathrm{U}$ relaxation calculations are performed with the ferromagnetic configuration since correlations are included by imposing a long-range magnetic order in $\mathrm{DFT}+\mathrm{U}$.

$\mathrm{LaCoO}_{3}$ is a rhombohedral structure with the $R \overline{3} c$ symmetry containing two Co ions per unit cell. The experimental crystal volume $V$ is $56.0 \AA^{3}$ per formula unit at low temperatures 17 . We find that the $\mathrm{DFT}+\mathrm{U}$ relaxation calculation with the PBE functional converging to LS produces the volume $V$ of $56.40 \AA^{3}$ per formula unit with the Co-O bond-length $a \sim 1.95 \AA$ (S1 structure in Table. (I), while the IS state (magnetic moment $=2.2 \mu B$ ) results in the $2.7 \%$ volume expansion resulting $V \sim$ $57.98 \AA^{3}$ per formula unit and $a \sim 1.97 \AA$ (S2 structure in Table. (I). The HS structure converged to a more expanded volume but the total energy is much higher than either LS or IS one, therefore we do not consider the HS structure in this paper. Interestingly, the MS imposing HS to one Co ion and LS to the other Co ion within the unit-cell produces the BD structure by lowering the crystal symmetry from $R \overline{3} c$ to $R \overline{3}$ (S3 structure in Table【. The crystal volume $V$ is similar to the IS volume $\left(V \sim 57.98 \AA^{3}\right)$ and the HS site (magnetic moment $\left.=3 \mu B\right)$ becomes a Co-O LB site with $a \sim 1.99 \AA$ and the LS site (magnetic moment $=0.3 \mu B$ ) is the SB with $a \sim 1.94 \AA$, resulting in the bond-length difference $\delta a \sim 0.05 \AA$. The $d$-occupancy $\left(N_{d}\right)$ for relaxation results in all structures are close to $d^{7}$ (leaving one hole in surrounding oxygen atoms) although the nominal $d$-occupancy is $d^{6}$ for $\mathrm{LaCoO}_{3}$. This is due to the strongly covalent nature of the $\mathrm{Co}-\mathrm{O}$ bonding in $\mathrm{LaCoO}_{3}$. The summary of the relaxed structure information is given in Table【. 


\section{B. DFT+DMFT}

Using LS, IS, and MS structures (denoted as S1, $\mathrm{S} 2$, and $\mathrm{S} 3$ in Table (I) obtained from DFT $+\mathrm{U}$ relaxations, we employ a charge-self-consistent DFT+DMFT method 42,43 to study the nature and energetics of the spin-state transition. The DFT+DMFT method is implemented using the maximally localized Wannier functions (MLWFs) $\stackrel{44,45}{4}$ as localized orbitals. First, we solve the non-spin-polarized Kohn-Sham (KS) equation within DFT using the VASP code. Then, we construct Co $3 d$ and $\mathrm{O} 2 p$ orbitals using MLWFs to represent the hybridization subspace for solving DMFT equations. The Co-O covalency effect can be treated within DMFT by including both $d$ and $p$ orbitals in the hybridization subspace. The $p-d$ Hamiltonian of the MLWF basis is constructed from the DFT bands in the hybridization energy window of $11 \mathrm{eV}$. Then, the correlated subspace of Co $3 d$ orbitals is treated using the continuous time quantum Monte Carlo (CTQMC) ${ }^{46,47}$ impurity solver by solving the DMFT self-consistent equations. The Hubbard $\mathrm{U}$ is $6 \mathrm{eV}$ and the Hund's coupling $\mathrm{J}$ is $0.9 \mathrm{eV}$ within the Co $3 d$ shell while we compute the local self-energy for Co $3 d$ orbitals within DMFT calculations for the study of spin-state transitions. Within DMFT, we use temperatures from $100 \mathrm{~K}$ to $1000 \mathrm{~K}$ to study the temperature effect on the spectral function while $300 \mathrm{~K}$ is used for most calculations unless specified otherwise. Here, both $\mathrm{U}$ and $\mathrm{J}$ are parameterized by Slater integrals $\left(F^{0}\right.$, $F^{2}$, and $\left.F^{4}\right)$, namely $\mathrm{U}=F^{0}$ and $\mathrm{J}=\left(F^{2}+F^{4}\right) / 14$. The Coulomb interaction matrix elements with only densitydensity types are considered in CTQMC while the spinflip and pair-hopping terms are neglected. Also, we chose the cartesian axes of Wannier orbitals to be aligned along the Co-O bonds so that the off-diagonal terms in the $d$-Hamiltonian can be much smaller (even zeros) than the on-site terms (see Appendix A). Therefore, the offdiagonal terms in the DMFT hybridization function can be also ignored within CTQMC.

The charge-self-consistency in DFT+DMFT can be achieved by updating the charge density using the DMFT local Green's function while the DMFT loop is converged. The KS equation is solved again using the updated charge density and the new $p-d$ Hamiltonian is constructed using the updated MLWFs obtained from the KS solutions. The full charge-self-consistent DFT+DMFT loop is continued until both the charge density, the DMFT Green's function $G^{l o c}$, and the DMFT self-energy $\Sigma^{l o c}$ are converged. More details of the DFT+DMFT implementation combining the projected augmented wave method in the DFT part and the formula for the charge update can be found in Ref. 42. For the precise convergence, the energy cut-off of $600 \mathrm{eV}$ and the $k$-point mesh of $8 \times 8 \times 8$ are used for DFT loops and a more dense $k$-point mesh of $30 \times 30 \times 30$ is used within the hybridization window of Wannier orbitals for DMFT loops.

Once the DFT+DMFT self-consistency loop is converged, the total energy $E$ is computed using the follow- ing formula:

$$
\begin{aligned}
E= & E^{D F T}[\rho]+\frac{1}{N_{\mathbf{k}}} \sum_{\mathbf{k}, i \in W} \epsilon_{i \mathbf{k}} \cdot\left(n_{i \mathbf{k}}-n_{i \mathbf{k}}^{0}\right) \\
& +E^{P O T}\left[G^{l o c}\right]-E^{D C}\left[N_{d}\right]
\end{aligned}
$$

where $E^{D F T}$ is the DFT energy computed using the charge density $\rho$ obtained within DFT $+\mathrm{DMFT}, \epsilon_{i \mathbf{k}}$ is the DFT eigenvalues, $n_{i \mathbf{k}}$ is the diagonal part of the DMFT occupancy matrix element with the KS band index $i$ and the momentum $\mathbf{k}, W$ is the energy window for the hybridization subspace, and $n_{i \mathbf{k}}^{0}$ is the DFT occupancy matrix element with the KS band $i$ and the momentum $\mathbf{k}$. The potential energy $E^{P O T}$ within DMFT is given by the Migdal-Galiski formula: $E^{p o t}=\frac{1}{2} \operatorname{Tr}\left[\Sigma^{l o c}(i \omega) \cdot G^{l o c}(i \omega)\right]$.

The double counting (DC) energy, $E^{D C}$ needs to be defined for beyond-DFT methods such as DFT+DMFT and DFT $+U$ since the potential energy treated in the correlated subspace is already accounted as the part of the DFT energy and it needs to be subtracted from the total energy formula. Various DFT+DMFT calculations suggest that the DC potential, $V^{D C}\left(=\partial E^{D C} / \partial N_{d}\right)$, smaller than the conventionally used fully-localized-limit (FLL) form $^{48}$ can produce better agreements of energetics 42,49 , the metal-insulator transition ${ }^{50}-52$, and the $p-d$ orbital splitting $50,52,53$ of oxides compared to experiments. Recently, it has been also shown that the more exact form of $V^{D C}$ within DFT+DMFT can be derived ${ }^{53}$ and the exact $V^{D C}$ value can be close to the nominal DC form, in which the $d$-occupancy, $N_{d}$, in the FLL formulae is replaced to the nominal $d$-occupancy, $N_{d}^{0}$ ( $d^{6}$ in the $\mathrm{LaCoO}_{3}$ case). More detailed discussions about different formula of double counting corrections are given in Appendix B.

In this paper, we propose the following form of $V^{D C}$ to allow the change between the FLL form and the nominal DC form by replacing $N_{d}$ to $\overline{N_{d}}$ :

$$
\begin{array}{r}
E^{D C}=\frac{U}{2} \cdot \overline{N_{d}} \cdot\left(\overline{N_{d}}-1\right)-\frac{J}{4} \cdot \overline{N_{d}} \cdot\left(\overline{N_{d}}-2\right) \\
V^{D C}=U \cdot\left(\overline{N_{d}}-\frac{1}{2}\right)-\frac{J}{2} \cdot\left(\overline{N_{d}}-1\right)
\end{array}
$$

where $\mathrm{U}$ and $\mathrm{J}$ are the same paramters which are defined above for the Slater-type interaction, $\overline{N_{d}}=N_{d}-\alpha$ where $N_{d}$ is the $d$-occupancy obtained self-consistently at each correlated site, and $\alpha$ is a parameter which can be tuned for obtaining different $E^{D C}$ and $V^{D C}$ values from the conventional FLL form. Our $V^{D C}$ formula can be derived from $E^{D C}\left(V^{D C}=\partial E^{D C} / \partial N_{d}\right)$ and allow sitedependent DC potentials. Our modified $V^{D C}$ form can recover the conventional FLL DC form by setting $\alpha=0$. By increasing $\alpha, V^{D C}$ approaches to the nominal $V^{D C}$ value as $\overline{N_{d}}$ becomes $N_{d}^{0}\left(\alpha=N_{d}-N_{d}^{0}\right)$. One should note that changing $V^{D C}$ with different $\alpha$ values can also tune the $p-d$ covalency effect by effectively shifting the $d$ orbital level. For example, a smaller $V^{D C}$ potential than the FLL DC potential will make the $d$ orbital level higher and the covalency effect weaker, resulting in a reduced $N_{d}$ value. The $p-d$ energy separation predicted by DFT or 
DFT $+\mathrm{U}$ with the FLL DC can be usually overbound and the physical role of $\alpha$ is to avoid this overbinding effectively by increasing the $p-d$ energy separation. In this paper, We studied the effect of the Co-O covalency on energetics and the nature of spin states in $\mathrm{LaCoO}_{3}$ by changing $V^{D C}$ potentials using different $\alpha$ values.

\section{RESULTS}

\section{A. Energetics of spin-state transition: $\mathrm{DFT}+\mathrm{DMFT}$ vs DFT+U}

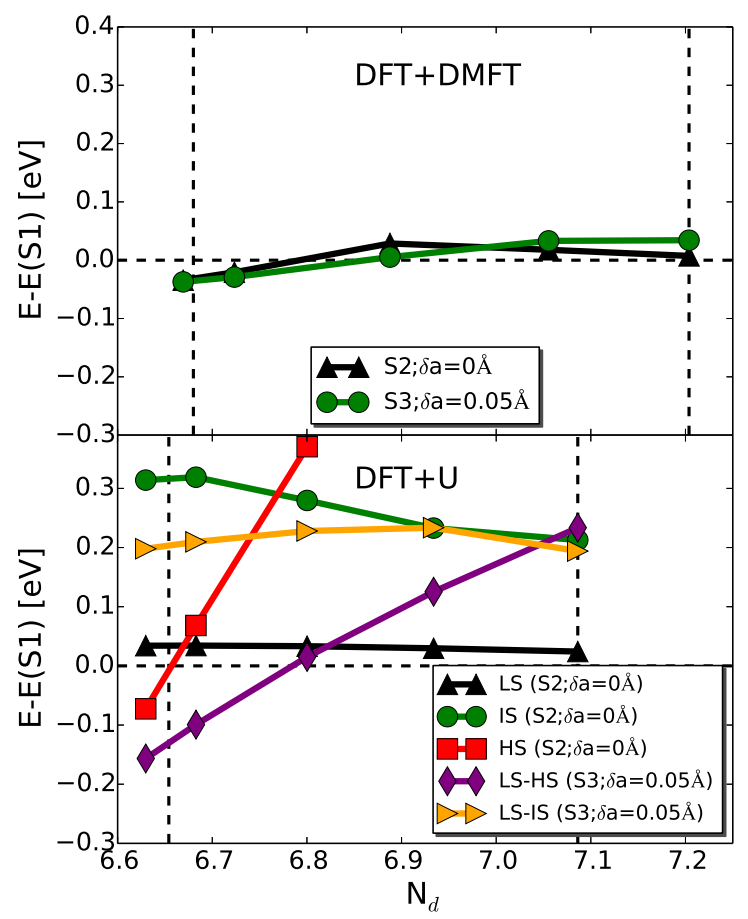

FIG. 1. Total energy differences between excited spin-state structures (S2 and S3) and the LS structure (S1) in $\mathrm{LaCoO}_{3}$ computed using DFT+DMFT (top panel) and DFT $+\mathrm{U}$ (bottom panel) as a function of $N_{d}$ tuned by different $\alpha$ values in $V^{D C}$ (Eq. 3). The S3 structure $(\delta \mathrm{a}=0.05 \AA)$ can incorporate the mixed spin-states between two Co ions in the unit cell. The left vertical dashed line represents the $N_{d}$ value obtained from the nominal DC formulae while the right dashed line shows $N_{d}$ obtained using the FLL DC formulae. Both DFT+DMFT and DFT $+\mathrm{U}$ methods use the same correlated orbitals (MLWFs) with the same interaction parameters $(\mathrm{U}=6 \mathrm{eV}$ and $\mathrm{J}=0.9 \mathrm{eV})$. Temperature is $300 \mathrm{~K}$ within DMFT calculations.

Here, we begin by showing the effect of different DC potential $\left(V^{D C}\right)$ values on the energetics of spin-states in $\mathrm{LaCoO}_{3}$ computed using DFT+DMFT (Fig.1 top panel) and DFT $+\mathrm{U}$ (Fig. 1 bottom panel). The $x$-axis shows $N_{d}$ values obtained by changing $V^{d c}$ in Eq. 3 using different $\alpha$ values, namely $\alpha=0,0.2,0.4,0.6$, and 0.7 . Two vertical dashed lines indicate the $N_{d}$ values obtained using the FLL DC formulae (the right line) and the nominal DC formulae (the left line). The calculations with the FLL DC $(\alpha=0)$ converge to $N_{d} \sim 7.2$ for DFT + DMFT and 7.1 for $\mathrm{DFT}+\mathrm{U}$ while those with the nominal DC result in $N_{d} \sim 6.68$ for DFT+DMFT and 6.65 for DFT $+\mathrm{U}$. The $y$-axis indicates the total energy difference between the expanded volume structures ( $\mathrm{S} 2$ and $\mathrm{S} 3 ; V \sim 57.98 \AA^{3}$ ) accompanying excited spin-states and the S1 structure $\left(V \sim 56.4 \AA^{3}\right)$. Here, DFT $+\mathrm{U}$ energies are computed by adopting the MLWFs as correlated orbitals consistently with DFT+DMFT calculations, therefore the difference between DFT $+\mathrm{DMFT}$ and $\mathrm{DFT}+\mathrm{U}$ results is attributed purely to the dynamical correlation effect beyond DFT $+\mathrm{U}$. Also, the previous study of $\mathrm{DFT}+\mathrm{U}$ calculations using different choices of orbitals (MLWFs vs projectors) has shown that results of the structural phase diagram of nickelates are almost the same as long as the same $U$ and $J$ values are used ${ }^{54}$. Therefore we expect that the energetics results reported here will not depend much on the choice of correlated orbitals.

The energetics obtained using two methods show noticeable differences depending on $N_{d}$ (the Co-O covalency effect). The DFT+DMFT energy difference between two expanded volume structures $(\mathrm{S} 2(\delta \mathrm{a}=0 \AA)$ and $\mathrm{S} 3(\delta \mathrm{a}=0.05 \AA)$ ) is much smaller (maximally $20 \mathrm{meV}$ ) than the $\mathrm{DFT}+\mathrm{U}$ energy difference while $\mathrm{DFT}+\mathrm{U}$ solutions converge to various meta-stable states for a given structure (LS, IS, and HS for S2 and LS-HS and LS-IS for S3) and the DFT $+\mathrm{U}$ energy depends sensitively on $N_{d}$ and spin-states. This difference in energetics arises since the spin-state within DFT+DMFT is described as a multiconfigurational state with a mixture of various spin states showing smooth crossover (see Fig.2) while the DFT+U solution is based on a single-determinant form and produces meta-stable states depending on given structure and $N_{d}$.

Within DFT+DMFT, the S2 structure (triangular dot) is more stable when $N_{d}>7.0$ and the LS state is dominant while the S3 BD structure (circular dot) becomes stable when the spin-state transition to LS-HS occurs $\left(N_{d}<7.0\right)$. Two structures become energetically almost the same when $N_{d}$ is further reduced $\left(N_{d} \sim 6.7\right)$ since the energy of the $\mathrm{S} 2$ structure gets lowered as HS is more excited at smaller $N_{d}$. The dependence of DFT+U energetics on $N_{d}$ favors the MS (LS-HS) state although it behaves qualitatively similar to DFT+DMFT as LS with the S2 structure is stable when $N_{d}>6.8$ and LSHS with the S3 BD structure becomes rapidly stable when $N_{d}$ is further reduced. The energy of HS with the S2 structure is also rapidly decreasing at smaller $N_{d}$ as the Hund's coupling lowers the energy in the HS state. Within DFT $+\mathrm{U}$, energetics of other meta-stable states including LS and LS-IS do not depend much on $N_{d}$ while IS is not favored as $N_{d}$ is reduced. Our calculations show that the spin-state transition from the S1 (LS) structure to excited spin-states with the expanded volume $(E-E(S 1)<0)$ occurs when $N_{d}$ becomes smaller $(<6.9$ for $\mathrm{DFT}+\mathrm{DMFT}$ and $<6.8$ for $\mathrm{DFT}+\mathrm{U})$ than 
the FLL DC result. This is consistent with the nominal $V^{D C}$ result (the left vertical dashed lines; $N_{d} \sim 6.68$ for DFT + DMFT) and due to the fact that higher spin states are more excited at smaller $N_{d}$ as will be shown in Fig.2,

\section{B. Nature of paramagnetic states}

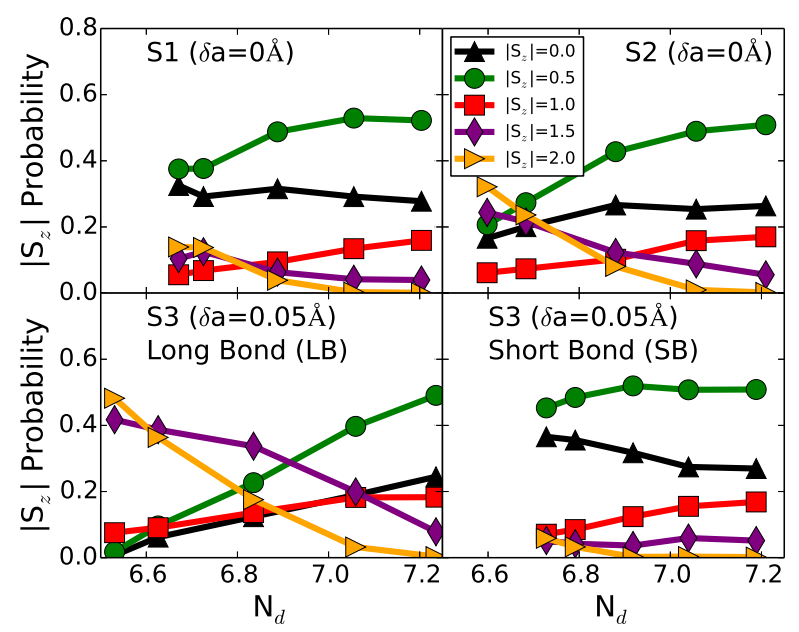

FIG. 2. The occupation probability of the spin $\left|S_{z}\right|$ state sampled using the CTQMC method within DFT+DMFT as a function of $N_{d}$ for the S1 structure $\left(V=56.40 \AA^{3}\right.$, upper left panel), the $\mathrm{S} 2$ structure $\left(V=57.98 \AA^{3}\right.$, upper right panel), and the S3 structure with the long-bond (lower left panel) and the short-bond (lower right panel) sites.

Now we turn to the nature of paramagnetic states in $\mathrm{LaCoO}_{3}$ obtained within DFT+DMFT. Fig. 2 displays the spin $\left|S_{z}\right|$ probabilities sampled using CTQMC as a function of $N_{d}$ for different structures. The multiconfigurational nature of the paramagnetic state treated in DFT+DMFT means that various $\left|S_{z}\right|$ states $\left(S_{z}=\right.$ $0.0 \sim 2.0)$ contribute to the solution. In all structures, the charge $d^{7}$ state with $\left|S_{z}\right|=0.5$ and 1.5 is not negligible in addition to the nominal $d^{6}$ state with $\left|S_{z}\right|=$ 0,1 , and 2 , therefore $\mathrm{LaCoO}_{3}$ is strongly covalent with the dynamically fluctuating nature of spin and charge states. When $N_{d}>7.0$, LS with $\left|S_{z}\right|=0$ and 0.5 has the highest probability for all structures, consistently with the fact that the $\mathrm{S} 1$ structure $\left(V=56.40 \AA^{3}\right)$ is energetically stable. As $N_{d}$ is reduced, LS is still dominant for the S1 structure while HS with $\left|S_{z}\right|=1.5$ and 2 becomes more excited for the $\mathrm{S} 2$ structure $\left(V=57.98 \AA^{3}\right)$. The nature of paramagnetic state in the $\mathrm{S} 2$ structure, which is energetically stable when $N_{d}<6.7$, is characterized by a mixture of both LS and HS, fluctuating dynamically with non-negligible probabilities. The IS state $\left(\left|S_{z}\right|=1.0\right)$ is strongly suppressed for all structures. In the BD structure, HS with $\left|S_{z}\right|=1.5$ and 2 becomes rapidly favored in the $\mathrm{LB}$ site as $N_{d}$ is reduced while LS with $\left|S_{z}\right|=0$ and 0.5 is always dominant in the SB site. These DFT+DMFT results of paramagnetic states ex- hibiting the mixture of different spin states are distinct from the DFT $+\mathrm{U}$ results where meta-stable solutions of spin states are found.

\section{Charge ordering induced by spin-state ordering}

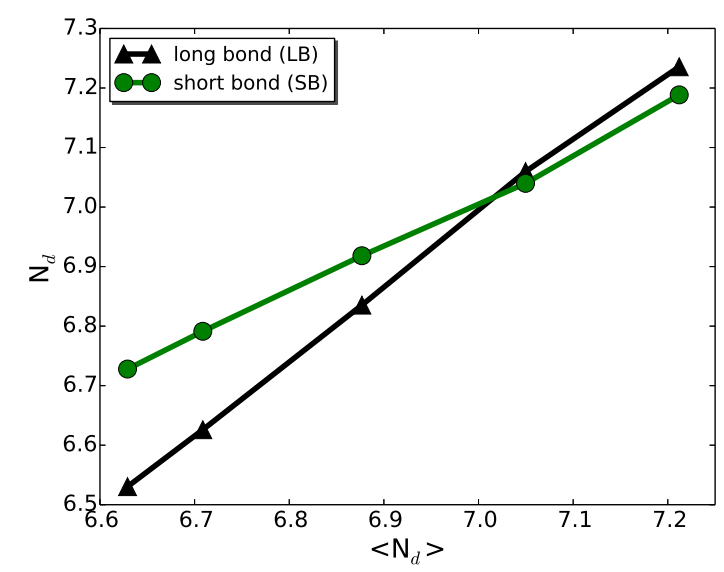

FIG. 3. The $d$-occupancy, $N_{d}$ computed for both the longbond and the short-bond sites in the S3 structure computed using DFT+DMFT as a function of the average $\left\langle N_{d}\right\rangle$ between two sites.

The strongly coupled spin and charge degrees of freedom also produce an intriguing rock-salt type charge ordering state induced from the MS state in the S3 structure. Fig. 3 shows that the DFT+DMFT charge in the LB site (triangular dots) gets smaller more rapidly than the SB charge (circular dots) as the average $\left\langle N_{d}\right\rangle$ becomes reduced (the overall Co-O covalency is reduced). This is because HS in the LB site favors the $\left|S_{z}\right|=2$ with $d^{6}$ state while the $\left|S_{z}\right|=0.5$ with $d^{7}$ state is dominant in the $\mathrm{SB}$ site as the $\mathrm{Co}-\mathrm{O}$ covalency remains strong. This enhancement of charge ordering when $N_{d}<6.9$ is also consistent with the rapid increase of the HS probability in the LB site as shown in Fig. 2. The experimental evidence of charge ordering with the concomitant spin-state ordering in $\mathrm{LaCoO}_{3}$ has been reported in the tensile-strained film ${ }^{23}$. The ground-state of this charge ordered $\mathrm{LaCoO}_{3}$ is insulating and the nature of the LB state is a Mott insulator while the SB state is a band insulator due to the strong Co-O hybridization (see Fig.8). This MS state in the S3 (BD) structure is somewhat reminiscent of the site-selective Mott physics occurring in nickelates in which the LB site is Mott insulating with the $d^{8}$ state while the SB site is a covalent insulator hybridized with $\mathrm{O}$ hole states as $d^{8} L^{2} .55$ Although charge ordering between two $\mathrm{Ni}$ sites in nickelates is not important to induce the insulating state in nickelates, charge ordering $(\sim 0.2)$ in $\mathrm{LaCoO}_{3}$ is naturally induced from the spin-state ordering. Also, nickelates are negative chargetransfer insulators meaning that almost one electron is donated to each $\mathrm{Ni}$ ion from surrounding $\mathrm{O}$ ions while 
the $\mathrm{Co}$ ion in $\mathrm{LaCoO}_{3}$ favors a mixed-valence state with $N_{d}=6.6 \sim 6.7$ as the charge transfer from the $\mathrm{O}$ hole is smaller than nickelates.

\section{Density of states}

In this subsection, we study the correlation, structure, $p-d$ covalency, and temperature effects on the spectral functions of $\mathrm{LaCoO}_{3}$. First, we show the density of states (DOS) computed using DFT paramagnetic calculations (no spin polarization) in Fig.4. The DOS computed for the different structures shows very similar features. All structures are metallic without correlations and the S1 structure has slightly larger band-width than other structures due to the smaller crystal volume. Co $t_{2 g}$ states are all occupied below the Fermi energy Co $e_{g}$ states are widely spread due to the strong mixing with the $\mathrm{O} p$ states. The LB and SB sites in the S3 structures exhibit the similar DOS without charge ordering $\left(N_{d} \sim 7.2\right)$.

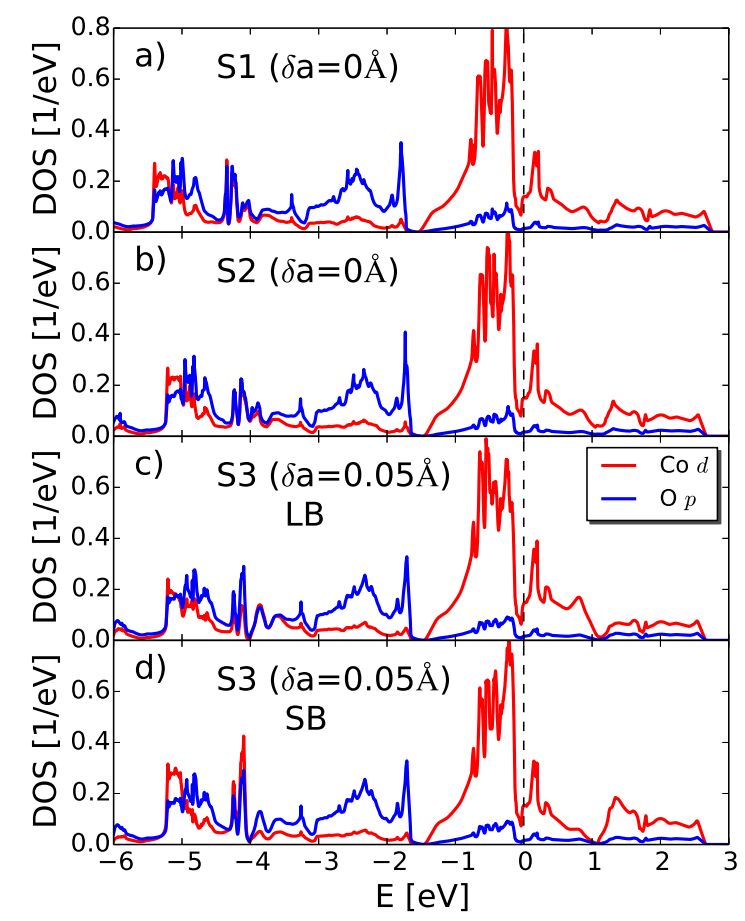

FIG. 4. The density of states computed for different structures of $\mathrm{LaCoO}_{3}$ using DFT with the paramagnetic (no spinpolarization) symmetry. They are a) $\mathrm{S} 1\left(V=56.40 \AA^{3}\right)$, b) S2 $\left(V=57.98 \AA^{3}\right)$, and $\mathrm{S} 3\left(V=57.98 \AA^{3}\right)$ structures with c) the Co-O long bond (LB) site and d) the short bond (SB) site.

Now, we include strong correlation effects in three structures (S1, S2, and S3) within DFT+DMFT and compute the DOS using the paramagnetic spin configuration in Fig. 5. We see dramatic changes of electronic structures due to correlation effects as well as the structural changes from the volume expansion and the Co-O bond disproportionation. The DOS in the S1 structure

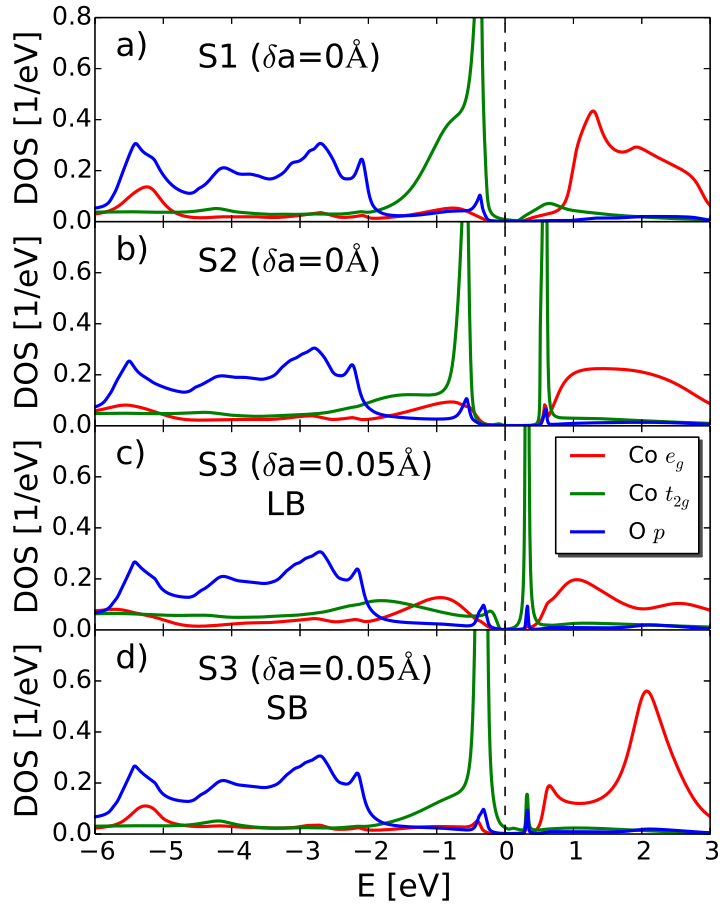

FIG. 5. The density of states obtained for different structures of $\mathrm{LaCoO}_{3}$ using DFT+DMFT with $\mathrm{U}=6 \mathrm{eV}, \mathrm{J}=0.9 \mathrm{eV}$, and the DC parameter $\alpha=0.7(\mathrm{Eq} \cdot 3)$ resulting $N_{d} \sim 6.68$. Different structures are a) $\mathrm{S} 1\left(V=56.40 \AA^{3}\right)$, b) S2 $\left(V=57.98 \AA^{3}\right)$, and $\mathrm{S} 3$ with c) the Co-O long bond and d) the short bond sites.

(Fig.5 a) shows that the band gap is almost $0.6 \mathrm{eV}$, which is consistent with the optical gap measurement $\underline{56}$. The $t_{2 g}$ state is almost occupied while the $e_{g}$ state is mostly unoccupied, as expected for the LS state. As the volume is expanded, the spin-state transition to higher spins occurs continuously and the $t_{2 g}$ state begins to be unoccupied while more $e_{g}$ orbitals are occupied (Fig $[5 \mathrm{~b})$. In the S1 structure, the major optical transition occurs from the Co $t_{2 g}$ valence band peak to the Co $e_{g}$ conduction band peak while the transition between the same $t_{2 g}$ bands dominates in the S2 structure. Therefore, the position of the first major peak in the optical conductivity will be reduced as the crystal volume expands at higher temperature, which is consistent with the optical conductivity measurement in experiment. Moreover, our calculation shows that the S3 structure with the MS state produces the smaller gap than the S2 structure, therefore the optical gap can be further reduced as temperature is raised presumably with more populated MS states. The nature of the paramagnetic insulating state in the $\mathrm{S} 2$ structure is a strongly correlated band insulator driven by both the Co-O hybridization due to the covalency and the electron localization of the Co d orbitals due to increased HS states. This physics is captured in the imaginary part of the self-energy (see Fig $8 \mathrm{~b}$ ) showing the emergence of the sharp pole outside the hybridization gap near the Fermi 
energy. This paramagnetic insulating state is represented as a mixture of fluctuating HS and LS states (see Fig.2) and it is distinct from the normal Mott insulator driven by the pure electron localization which is expected from the HS state in a d6 configuration. In the S3 structure, the LB site becomes higher spin state with more unoccupied $t_{2 g}$ states while the SB site remains as LS with a similar gap size as the homogeneous LS gap. Here, the $\alpha$ value in $V^{D C}$ (Eq. 3 ) is set to 0.7 resulting $N_{d} \sim 6.68$ for all structures. Different $V^{D C}$ values will change the relative position of the $\mathrm{O} p$ peak from the Fermi energy as $N_{d}$ is also varied. Our $\mathrm{O} p$ top peak position is located at $-2 \mathrm{eV}$ when $N_{d} \sim 6.68$ and this peak position is consistent with the measured X-ray photo-emission spectra ${ }^{4}$ validating the $\alpha$ value we used.

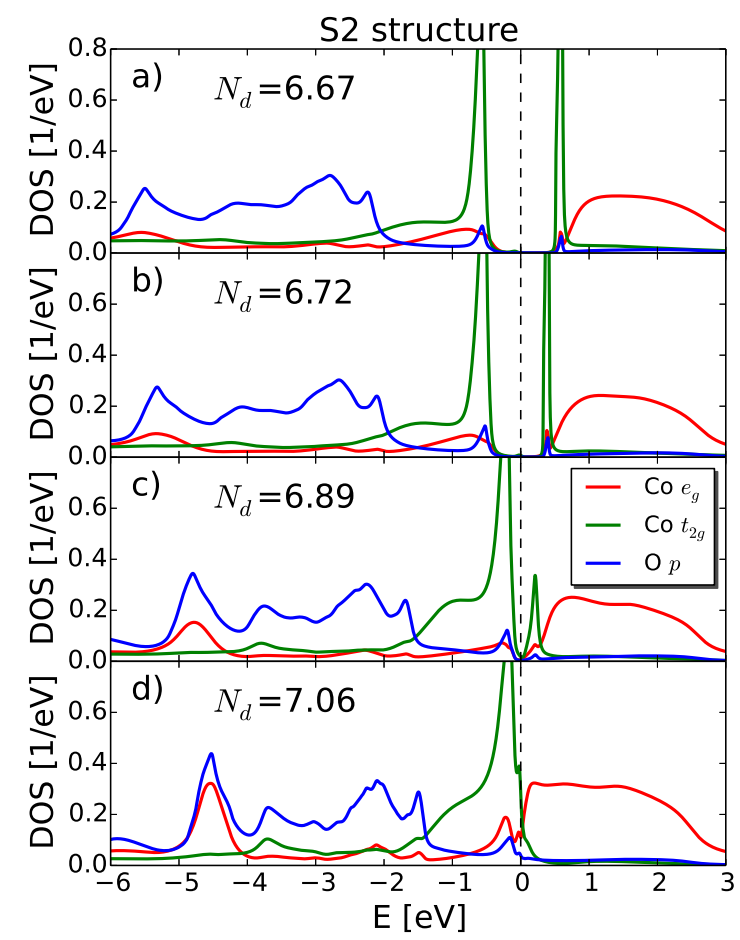

FIG. 6. The density of states computed using different $\alpha$ values in Eq.3 within DFT+DMFT. The S2 structure is used. Different $\alpha$ values lead to distinct $N_{d}$ results, namely a) $N_{d}=$ $6.67(\alpha=0.7)$, b) $N_{d}=6.72(\alpha=0.6)$, c) $N_{d}=6.89(\alpha=$ $0.4)$, and $N_{d}=7.06(\alpha=0.2)$. $\mathrm{U}=6 \mathrm{eV}$ and $\mathrm{J}=0.9 \mathrm{eV}$ are used within DMFT.

To clarify the role of the Co-O covalency (parametrized by $N_{d}$ ) on electronic structure, we show the DOS obtained using different $\alpha$ values for the $\mathrm{S} 2$ structure in Fig. 6. As the Co-O covalency is enhanced $\left(N_{d}\right.$ is increased), the $\mathrm{O} p$ peaks move closer to Co $d$ states near the Fermi energy. As a result, the spectral gap becomes smaller due to the larger $\mathrm{Co}-\mathrm{O}$ hybridization and the ground-state is eventually metallic when $N_{d} \sim 7.1$. Therefore, the insulator-to-metal transition occurs as the correlation in the Co ion is reduced due to the increased Co-O covalency and, at the same time, the spin states are less excited as Co $t_{2 g}$ states are more occupied and $e_{g}$ states are more unoccupied. This DOS result is also consistent with the occupation probability data of the S2 structure (Fig.2 upper right panel) showing that the HS states $\left(\left|S_{z}\right|=1.5\right.$ and 2.0) are suppressed and the LS and IS states $\left(\left|S_{z}\right|=0.0,0.5\right.$, and 1.0) are gradually increasing as $N_{d}$ increases. Our results suggest that the Co-O hybridization due to the covalency can play an important role in explaining the metal-insulator transition and the spin-state transition in $\mathrm{LaCoO}_{3}$.

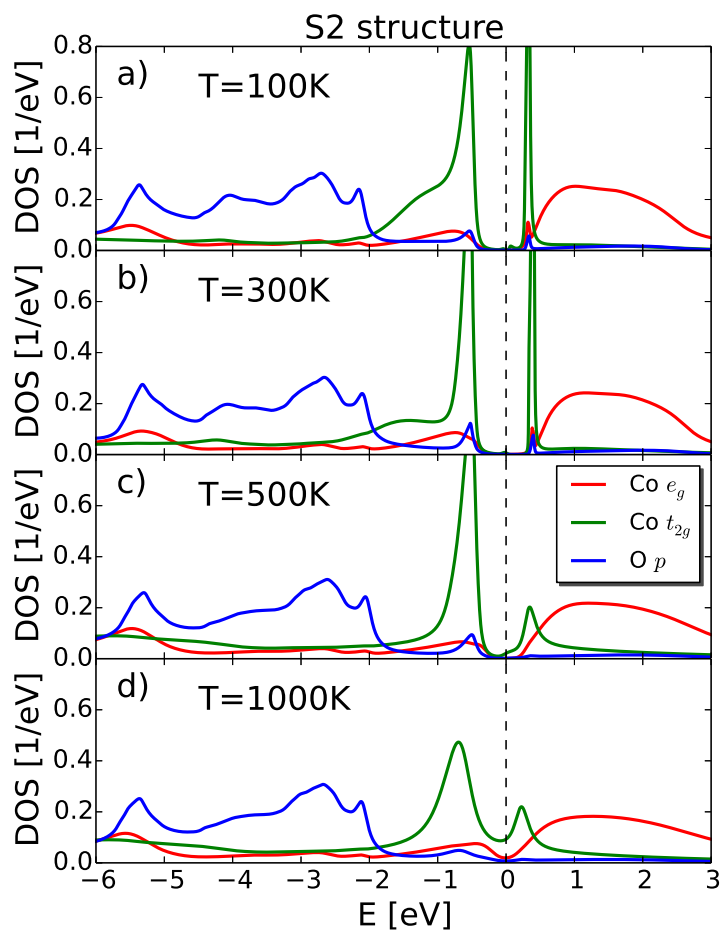

FIG. 7. The density of states computed for the S2 structure of $\mathrm{LaCoO}_{3}$ using DFT+DMFT at different temperatures, namely a) $T=100 K$, b) $300 K$, c) $500 K$, and d) $1000 K$. U $=6 \mathrm{eV}, \mathrm{J}=0.9 \mathrm{eV}$, and the double counting $\alpha=0.6$ $\left(N_{d} \sim 6.7\right)$ are used within DMFT.

Until now, our DFT+DMFT calculations have been performed at the fixed temperature $(\sim 300 K)$. Experimentally, $\mathrm{LaCoO}_{3}$ also exhibits the insulator-to-metal transition as temperature is raised above near $T=400 \mathrm{~K}$ but the nature of this transition has not been clarified. To better understand the role of temperature on the metal-insulator transtion in $\mathrm{LaCoO}_{3}$, we plot the DOS of $\mathrm{LaCoO}_{3}$ at the fixed S2 structure and different temperatures (Fig. 7). The DOS data computed at both $100 K$ and $300 K$ show similar features although the spectral gap at $300 K$ is slightly larger than one at $100 K$. The similarity of the DOS at low temperatures indicates that our spin-state calculations at $300 K$ can be similarly reproduced at $100 \mathrm{~K}$ (the experimental $T_{c}$ of the spinstate transition). More importantly, the strong variation of the DOS and spin-states depending on structures in Fig.5. 5 means that the structural changes should be incor- 
porated for the better description of electronic structure while temperatures are varied. Nevertheless, the spectral gap becomes smaller as temperature is raised above $500 K$ and the Co $t_{2 g}$ state becomes the incoherent metallic state at around $1000 \mathrm{~K}$.

\section{E. Self-energy data}
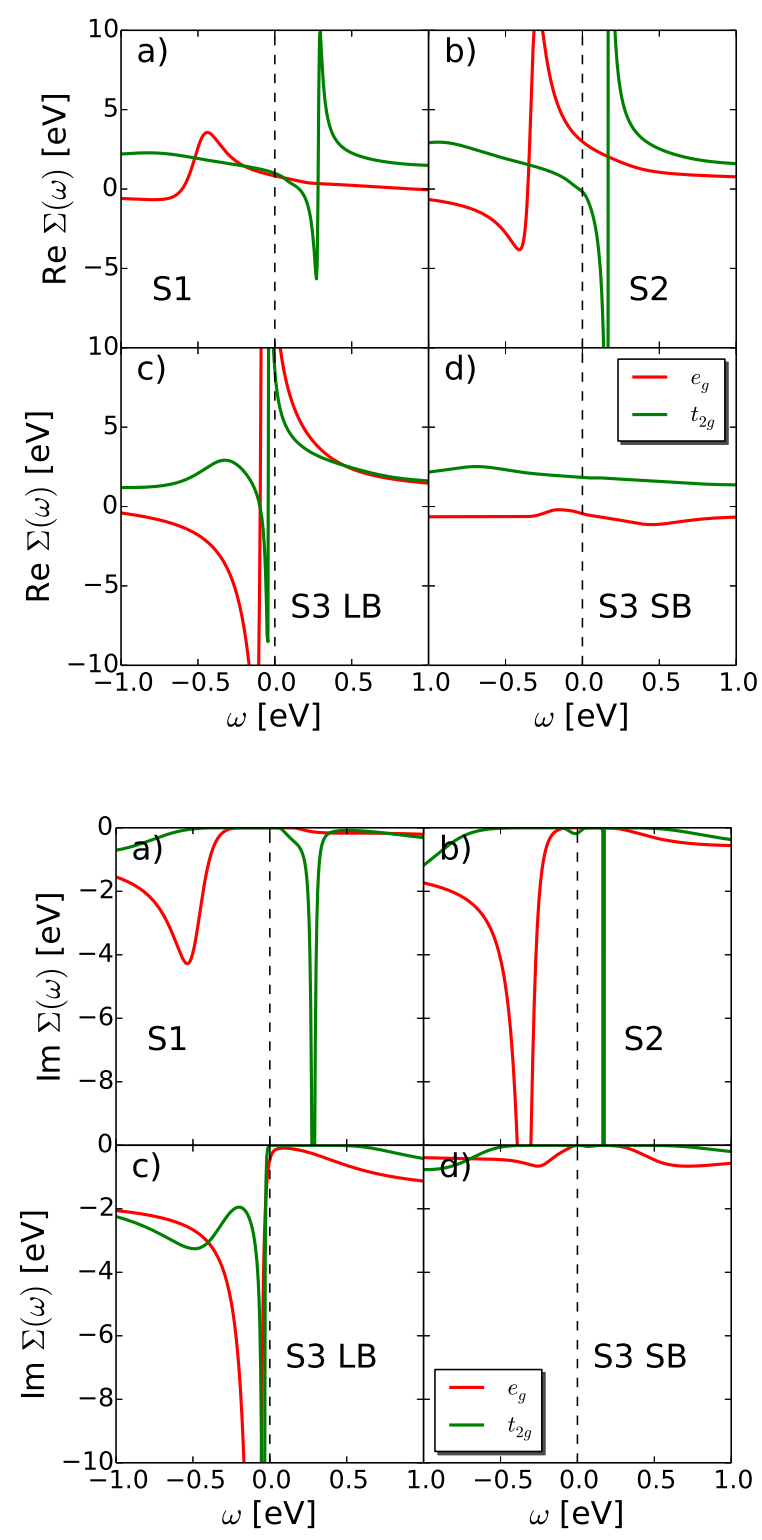

FIG. 8. The real part (top panel) and the imaginary part (bottom panel) of self-energy data computed for different structures in $\mathrm{LaCoO}_{3}$, namely a) S1, b) S2, c) S3 Co-O long bond (LB), and d) S3 Co-O short bond (SB). $\mathrm{U}=6 \mathrm{eV}, \mathrm{J}=0.9 \mathrm{eV}$, and the double counting parameter $\alpha=0.6$ $\left(N_{d} \sim 6.7\right)$ are used.

To study the nature of insulating states occuring in different stuctures, we show both the real part (Re $\Sigma$; Fig. 8 top panel) and the imaginary part (Im $\Sigma$; Fig. 8 bottom panel) of self-energies on the real axis, which are used to compute the DOS in Fig. 5. The S1 structure (Fig. 8a) exhibits rather small $\operatorname{Im} \Sigma$ values in both $e_{g}$ and $t_{2 g}$ orbitals when $\omega \sim 0$ while a sharp pole develops at $\omega \sim 300 \mathrm{meV}$ in the $t_{2 g}$ orbital for both real and imaginary parts. This diverging nature of the selfenergy indicates Co $d$ orbitals are still correlated even at the S1 structure with LS. This self-energy nature of LS is different from the typical band insulating nature of LS which is expected in a $d^{6}$ configuration. The nature of our insulating state is the correlated band insulator driven by both the Co-O hybridization and the electron correlation. The electron correlation is encoded in the sharp and narrow pole structure of the imaginary part of the self-energy developed outside the hybridization gap while the imaginary part of the self-energy is still zero at the Fermi energy. As the structure changes from S1 to $\mathrm{S} 2$ along with the volume expansion, the strength of poles becomes enhanced and the positions of the poles get close to the Fermi energy in both $e_{g}$ and $t_{2 g}$ orbitals. This clearly shows that correlations are enhanced due to the increased higher spin probabilities (see Fig.2) as the volume is expanded in $\mathrm{LaCoO}_{3}$. The insulating nature of the S3 structure shows the "site-selective" Mott physics, as the Co-O LB site undergoes a Mott transition with the diverging self-energies at the Fermi energy while the SB site behaves as a band insulator with the small imaginary part and the flat real part of self-energies originated from the strong $\mathrm{Co}-\mathrm{O}$ covalency.

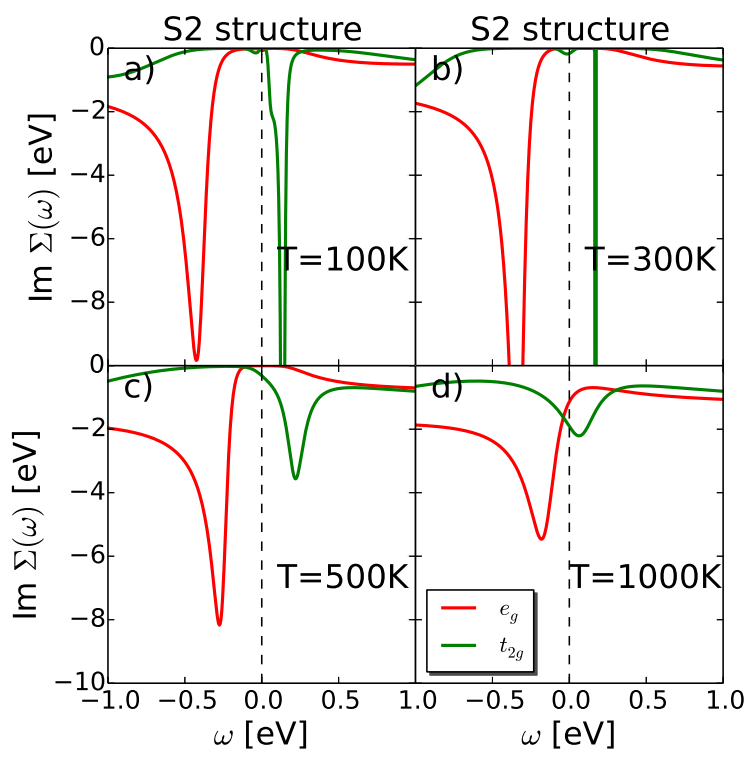

FIG. 9. The imaginary part of self-energy data computed for different temperatures at a fixed S2 structure in $\mathrm{LaCoO}_{3}$, namely a) $T=100 \mathrm{~K}$, b) $T=300 \mathrm{~K}$, c) $T=500 \mathrm{~K}$, and d) $T=1000 \mathrm{~K}$. U $=6 \mathrm{eV}, \mathrm{J}=0.9 \mathrm{eV}$, and the double counting parameter $\alpha=0.6\left(N_{d} \sim 6.7\right)$ are used. 
To understand the nature of the insulator-to-metal transition in $\mathrm{LaCoO}_{3}$ as temperature is raised, we also plot the $\operatorname{Im} \Sigma$ computed for the S2 structure as a function of temperature in Fig.9. Results of $\operatorname{Im} \Sigma$ at $T=100 \mathrm{~K}$ show that poles are developed due to correlations at $\omega=$ $-450 \mathrm{meV}$ for the $e_{g}$ orbital and at $\omega=150 \mathrm{meV}$ for the $t_{2 g}$ orbital. The strengths of these poles are enhanced at $T=300 \mathrm{~K}$ as the $\mathrm{S} 2$ structure can occupy higher spin states due to the spin-state transition and electron localization can be enhanced at this temperature. This physics is different from the typical Mott insulator without any spin-state transition, in which the electron localization is usually stronger at lower temperature. As the temperature is raised even above $300 \mathrm{~K}$, the pole strengths are reduced and correlations become weaker for both $e_{g}$ and $t_{2 g}$ orbitals resulting the metallic phase obtained in Fig. 7.

\section{F. Charge-self-consistency effect}

Finally, we show the charge-self-consistency effect in DFT + DMFT on the energetics and electronic structure in $\mathrm{LaCoO}_{3}$. Fig. 10 shows the energetics of spin-state transition in $\mathrm{LaCoO}_{3}$ as a function of $N_{d}$ comparing charge-self-consistent (top panel) and non-charge-selfconsistent (bottom panel) DFT+DMFT calculations. Here, the non-charge-self-consistent calculation means that the charge density ( $\rho$ in Eq. 1) is fixed to the DFT one while the DMFT local Green's function $\left(G^{l o c}\right.$ in Eq1) is obtained by converging DMFT self-consistent equations. As a result, the $V^{D C}$ potential is fixed during the DFT+DMFT loop since the charge-density is not updated and $V^{D C}$ is a function of the charge-density $\left(N_{d}\right)$. Therefore, different $N_{d}$ results in non-charge-selfconsistent DFT+DMFT (bottom panel) are obtained by shifting the $V^{D C}$ potential as data points are changed. However, in charge-self-consistent DFT+DMFT (top panel), $V^{D C}$ is computed using the Eq. 3 with the selfconsistently determined charge density $\left(N_{d}\right)$ and the corresponding $N_{d}$ is obtained from the DMFT Green's function.

The energetics of the S2 structure (homogeneous spin states) show the very similar behavior as a function of $N_{d}$ between charge-self-consistent and non-charge-selfconsistent calculations. In the case of the MS state for the S3 structure, non-charge-self-consistent DFT+DMFT energetics overestimate the tendency toward the spinstate ordering as the energy difference between the excited spin-state and the low-spin (S1) state becomes much lower $(\sim-150 \mathrm{meV})$ than the charge-self-consistent energetics $(\sim-30 \mathrm{meV})$ when $N_{d} \sim 6.7$. The energetics of the MS state also depend on $N_{d}$ much sensitively in the non-charge-self-consistent case. Therefore, the main effect of charge-self-consistency in DFT+DMFT is to reduce the spin-state ordering effect in the S3 structure and the energetics between homogeneous and MS spin-states in the S2 and S3 structures become very close.
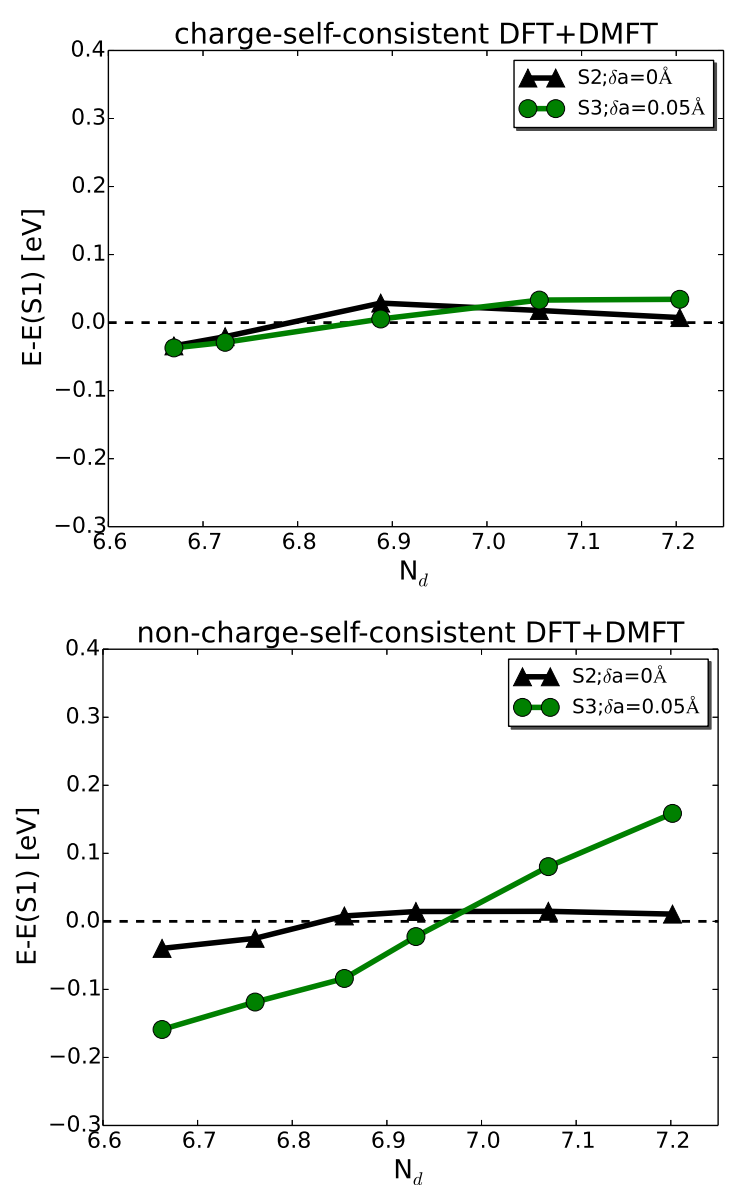

FIG. 10. Total energy differences between excited spin states ( $\mathrm{S} 2$ and $\mathrm{S} 3$ ) and the low spin state $(\mathrm{S} 1)$ in $\mathrm{LaCoO}_{3}$ computed using both charge-self-consistent DFT+DMFT (top panel) and non-charge-self-consistent DFT+DMFT (bottom panel) as a function of $N_{d}$ tuned by different $V^{D C}$ potentials. The excited spin states incorporates the volume expansion with or without the Co-O bond-disproportionation ( $\delta \mathrm{a}=0.05 \AA$ or $0 \AA)$. $\mathrm{U}=6 \mathrm{eV}, \mathrm{J}=0.9 \mathrm{eV}$, and temperature $\mathrm{T}=300 \mathrm{~K}$ are used within DMFT.

To further investigate the effect of charge-selfconsistency on electronic structure of $\mathrm{LaCoO}_{3}$, we display the $d$-occupancy, $N_{d}$ in Fig.11 obtained for both LB and $\mathrm{SB}$ sites in the S3 structure computed using charge-selfconsistent DFT+DMFT (solid lines) and non-charge-selfconsistent DFT+DMFT (dashed lines) as a function of the average $N_{d}$ between two sites. Without the charge update (dashed lines), the $N_{d}$ difference between two sites is more enhanced since the $\mathrm{SB}$ site occupies more $d$-orbitals while the LB site takes even less $N_{d}$ compared to the charge-self-consistent results (solid lines) across different $\left\langle N_{d}\right\rangle$ values. Therefore, charge-self-consistency within DFT+DMFT reduces the tendency toward both spin-state and charge orderings between correlated Co sites, as a result, the energy difference between different excited spin-states is also much decreased within the charge-self-consistent calculation. 


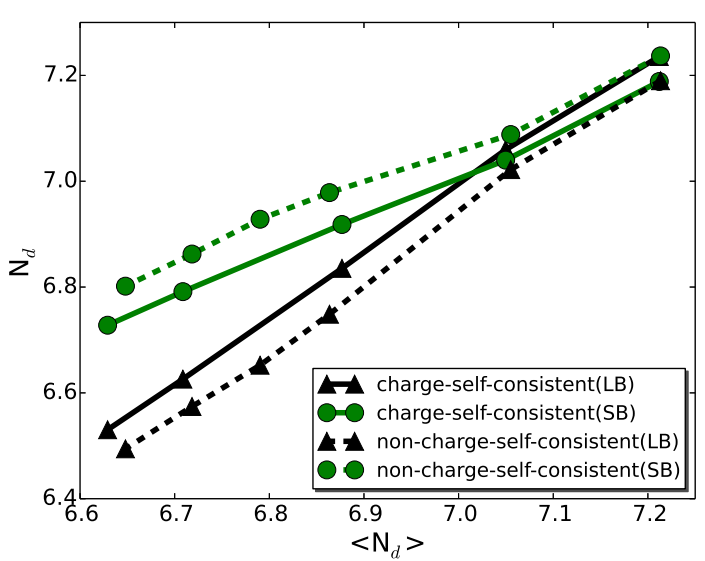

FIG. 11. The $d$-occupancy, $N_{d}$ computed for both LB and $\mathrm{SB}$ sites in the S3 structure computed using chargeself-consistent DFT+DMFT (solid lines) and non-charge-selfconsistent DFT+DMFT (dashed lines) as a function of the average $\left\langle N_{d}\right\rangle$ between two sites.

Here, we fix the $\mathrm{U}$ and $\mathrm{J}$ values during the DFT+DMFT calculations, therefore the main difference between charge-self-consistent and non-charge-selfconsistent results is originated from the change of the Wannier Hamiltonian due to the charge update (see Appendix A). The main role of the charge update is to decrease the crystal field splitting in the S1 and S2 structures and to promote the homogeneous spin-state transition. At the same time, the spin-state and charge ordering effects in the S3 structure has been reduced as the crystal field splitting in the LB site has been increased while it has been decreased in the SB site acting counterintuitively on the structural effect. Moreover, the overall $d$-orbital level in the LB site is substantially lowered than the level in the SB site due to the charge update, again decreasing the charge ordering effect. Therefore, the charge-self-consistent effect compensates for the spinstate and charge ordering effects in the S3 structure while it favors the homogeneous spin-state transition, and as a result, the energy difference between the spin-state ordering and the homogeneous spin state has been much reduced within the charge-self-consistent DFT + DMFT calculations.

\section{CONCLUSION}

In conclusion, we adopt the charge-self-consistent DFT+DMFT method to study the nature and energetics of both homogeneous and MS states in covalent $\mathrm{LaCoO}_{3}$. We find that structural changes during the spin-state transition are important to understand both energetics and electronic structure in $\mathrm{LaCoO}_{3}$. As the crystal volume is expanded, the occupation probability of higher spin state in a Co ion increases and a sharp and narrow peak in the imaginary part of the self-energy develops outside the Co-O hybridization gap due to the enhanced electron correlation. This paramagnetic insulating state also exhibit a multi-configurational mixture of both HS and LS states with strong spin and charge fluctuations. This DMFT result is different from the static DFT $+\mathrm{U}$ result showing various meta-stable solutions. The MS state in the $\mathrm{BD}$ structure accompanies the HS state in a LB Co site and the LS state in a SB site. Charge ordering is also induced from this spin-state ordering since HS favors the $d^{6}$ charge state while LS occupies more $d^{7}$ states as covalently bonded with $\mathrm{O}$ ions. The LB site with HS becomes a Mott insulator while the SB site with LS behaves as a band insulator. Our DFT+DMFT calculation reveals that energetics between homogeneous and MS states are very close while DFT $+\mathrm{U}$ energetics depend sensitively on spin-states and structures favoring the MS state.

We also find that the Co-O covalency plays a crucial role in electronic structure of $\mathrm{LaCoO}_{3}$. Changing the double-counting potential in DFT + DMFT can lead to a different $N_{d}$ value tuning the covalency effectively and both energetics and one-particle spectra are more consistent with experiments when $N_{d} \sim 6.7$. Increasing the $\mathrm{Co}-\mathrm{O}$ covalency produces the insulator-tometal transition faving the LS state. Increasing temperature beyond $500 \mathrm{~K}$ also reduces the correlation effect and drives the insulator-to-metal transition, similarly as the experiment. The charge-self-consistency effect within DFT+DMFT reduces the tendency toward spin-state and charge orderings in $\mathrm{LaCoO}_{3}$ producing close energetics between different structures compared to the non-charge-self-consistent calculation.

\section{ACKNOWLEDGMENTS}

H. Park acknowledges helpful discussions with Andrew Millis. H. Park and R. Nanguneri are supported by the U.S. Deptartment of Energy (DOE), Office of Science, Basic Energy Sciences (BES), Materials Sciences and Engineering Division. A. Ngo is supported by the Vehicle Technologies Office (VTO), Department of Energy (DOE), USA. We also gratefully acknowledge the computing resources provided on Bebop, a high-performance computing cluster operated by the Laboratory Computing Resource Center at Argonne National Laboratory.

\section{APPENDIX A: THE $d$-ORBITAL HAMILTONIAN}

In this Appendix, we show the matrix elements of the multi-orbital Hamiltonian, $\hat{H}^{d d}$, in the Co $3 d$ shell at each correlated site for three structures (S1, S2, S3 LB, and S3 SB) used in the DFT+DMFT calculations of this paper. First, we provide $\hat{H}^{d d}$ (in units of $\mathrm{eV}$ ) represented using MLWF $d$-orbitals obtained from the DFT solution, which is relevant to the non-charge-self-consistent 
DFT + DMFT calculation. The orbital order representing $\hat{H}^{d d}$ is $d^{3 z^{2}-r^{2}}, d^{x z}, d^{y z}, d^{x^{2}-y^{2}}$, and $d^{x y}$.

$$
\begin{aligned}
\hat{H}_{S 1}^{d d}= & \left(\begin{array}{ccccc}
6.912 & 0.006 & -0.05 & -0.00 & -0.01 \\
0.006 & 6.236 & 0.019 & -0.01 & -0.02 \\
-0.05 & 0.019 & 6.241 & -0.02 & -0.02 \\
-0.00 & -0.01 & -0.02 & 6.917 & -0.01 \\
-0.01 & -0.02 & -0.02 & -0.01 & 6.237
\end{array}\right) \\
\hat{H}_{S 2}^{d d}= & \left(\begin{array}{cccccc}
6.575 & -0.01 & -0.050 & -0.00 & 0.009 \\
-0.01 & 5.948 & -0.02 & 0.013 & -0.02 \\
-0.050 & -0.02 & 5.954 & -0.02 & 0.020 \\
-0.00 & 0.013 & -0.02 & 6.579 & 0.010 \\
0.009 & -0.02 & 0.020 & 0.010 & 5.949
\end{array}\right) \\
\hat{H}_{S 3, L B}^{d d}= & \left(\begin{array}{llllll}
6.484 & 0.003 & 0.054 & -0.00 & 0.005 \\
0.003 & 5.913 & -0.02 & -0.00 & 0.018 \\
0.054 & -0.02 & 5.921 & 0.027 & -0.02 \\
-0.00 & -0.00 & 0.027 & 6.489 & 0.002 \\
0.005 & 0.018 & -0.02 & 0.002 & 5.915
\end{array}\right) \\
\hat{H}_{S 3, S B}^{d d}= & \left(\begin{array}{llllll}
6.583 & 0.006 & 0.049 & -0.00 & 0.014 \\
0.006 & 5.909 & -0.02 & -0.02 & 0.022 \\
0.049 & -0.02 & 5.914 & 0.013 & -0.02 \\
-0.00 & -0.02 & 0.013 & 6.587 & 0.012 \\
0.014 & 0.022 & -0.02 & 0.012 & 5.910
\end{array}\right)
\end{aligned}
$$

First of all, the local axis for initial projections of MLWFs are chosen to be aligned to the Co-O octahedron axis, therefore the off-diagonal elements are close to zeros. The crystal-field splittings between $e_{g}$ and $t_{2 g}$ orbitals are $\sim 0.67 \mathrm{eV}$ for $\mathrm{S} 1, \sim 0.63 \mathrm{eV}$ for $\mathrm{S} 2, \sim 0.57 \mathrm{eV}$ for S3 LB, and $\sim 0.68 \mathrm{eV}$ for S3 SB. As expected, the Co$\mathrm{O}$ bond length can tune the crystal-field splitting (the energy difference between $e_{g}$ and $t_{2 g}$ orbitals). Namely, the long-bond Co ion has the smaller splitting and favors higher spin-states.

The charge-self-consistency effect in DFT+DMFT produces a new charge density $\rho$ which is different from the original DFT $\rho$. As a result, $\hat{H}^{d d}$ is computed from the updated $\rho$ and is also changed. Here, we show below $\hat{H}^{d d}$ obtained from charge-self-consistent DFT+DMFT calculations using $\mathrm{U}=6 \mathrm{eV}, \mathrm{J}=0.9 \mathrm{eV}$, and the $\mathrm{DC}$ parameter $\alpha=0.6$. In this case, the crystal-field splittings are $\sim 0.6 \mathrm{eV}$ for $\mathrm{S} 1$ and $\mathrm{S} 2, \sim 0.66 \mathrm{eV}$ for $\mathrm{S} 3 \mathrm{LB}$, and $\sim 0.54 \mathrm{eV}$ for $\mathrm{S} 3 \mathrm{SB}$. The main effect of the charge-selfconsistency on $\hat{H}^{d d}$ is to reduce the crystal-field splittings for S1 and S2 structures compared to the non-chargeself-consistency case, therefore it promotes the homogeneous spin-state transition. However, the charge-selfconsistency effect also compensates for the spin-state and charge orderings as the crystal-field splitting has been increased for the LB site while it is decreased for the SB site in the $\mathrm{S} 3$ structure. The average $d$-orbital level for the LB site becomes also much lower than the SB site, again compensating for charge ordering.

$$
\begin{aligned}
\hat{H}_{S 1}^{d d}= & \left(\begin{array}{ccccc}
4.332 & -0.01 & -0.01 & -0.00 & 0.014 \\
-0.01 & 3.737 & -0.01 & 0.022 & 0.006 \\
-0.01 & -0.01 & 3.737 & -0.02 & 0.004 \\
-0.00 & 0.022 & -0.02 & 4.333 & 0.022 \\
0.014 & 0.006 & 0.004 & 0.022 & 3.735
\end{array}\right) \\
\hat{H}_{S 2}^{d d}= & \left(\begin{array}{cccccc}
3.767 & 0.013 & -0.01 & -0.00 & -0.02 \\
0.013 & 3.158 & 0.006 & -0.02 & 0.007 \\
-0.01 & 0.006 & 3.157 & -0.02 & -0.01 \\
-0.00 & -0.02 & -0.02 & 3.767 & -0.02 \\
-0.02 & 0.007 & -0.01 & -0.02 & 3.156
\end{array}\right) \\
\hat{H}_{S 3, L B}^{d d}= & \left(\begin{array}{cccccc}
3.150 & -0.02 & 0.016 & -0.00 & -0.02 \\
-0.02 & 2.490 & 0.009 & 0.037 & -0.01 \\
0.02 & 0.009 & 2.488 & 0.033 & 0.006 \\
-0.00 & 0.037 & 0.033 & 3.148 & -0.04 \\
-0.02 & -0.01 & 0.006 & -0.04 & 2.487
\end{array}\right) \\
\hat{H}_{S 3, S B}^{d d}= & \left(\begin{array}{cccccc}
4.519 & -0.01 & 0.006 & -0.00 & -0.01 \\
-0.01 & 3.984 & 0.006 & 0.017 & -0.01 \\
0.006 & 0.006 & 3.984 & 0.014 & 0.005 \\
-0.00 & 0.017 & 0.014 & 4.520 & -0.02 \\
-0.01 & -0.01 & 0.005 & -0.02 & 3.983
\end{array}\right)
\end{aligned}
$$

\section{APPENDIX B: DOUBLE COUNTING CORRECTION}

A frequently used expression of the double-counting energy $E^{D C}$ is the fully localized limit (FLL) form 48 which has been adopted frequently in DFT $+\mathrm{U}$.

$$
\begin{aligned}
E^{D C} & =\frac{U}{2} \cdot N_{d} \cdot\left(N_{d}-1\right)-\frac{J}{4} \cdot N_{d} \cdot\left(N_{d}-2\right) \\
V^{D C} & =\frac{\partial E^{D C}}{\partial N_{d}}=U \cdot\left(N_{d}-\frac{1}{2}\right)-\frac{J}{2} \cdot\left(N_{d}-1\right)
\end{aligned}
$$

where $N_{d}$ is the occupancy of the correlated site and can be obtained as the result of self-consistent DFT + DMFT or $\mathrm{DFT}+\mathrm{U}$ calculations. Therefore, the $V^{D C}$ potential depends on the correlated site since $N_{d}$ is site-dependent.

Recently, it has been shown that the exact form of $V^{D C}$ within DFT+DMFT ${ }^{53}$ can be computed and the formulae should be close to the nominal DC form, where $N_{d}$ in Eq.13 is replaced to the nominal $d$-occupancy in the atomic limit, $N_{d}^{0}$, which is site-independent.

$$
V^{D C}=U \cdot\left(N_{d}^{0}-\frac{1}{2}\right)-\frac{J}{2} \cdot\left(N_{d}^{0}-1\right)
$$

The hybridization of $d$ and $p$ orbitals in transition metal oxides means that the resulting $d$-occupancy $N_{d}$ will be larger than the nominal value $N_{d}^{0}\left(N_{d}>N_{d}^{0}\right)$, therefore the nominal $V^{D C}$ will be always smaller than the FLL $V^{D C}$. This smaller $V^{D C}$ potential reduces the covalency effect between $d$ and $p$ orbitals. 
In this paper, we use the following forms of $E^{D C}$ and $V^{D C}$ by modifying the FLL forms to allow the tuning of $V^{D C}$ for changing the Co-O covalency effect.

$$
\begin{array}{r}
E^{D C}=\frac{U}{2} \cdot \overline{N_{d}} \cdot\left(\overline{N_{d}}-1\right)-\frac{J}{4} \cdot \overline{N_{d}} \cdot\left(\overline{N_{d}}-2\right) \\
V^{D C}=U \cdot\left(\overline{N_{d}}-\frac{1}{2}\right)-\frac{J}{2} \cdot\left(\overline{N_{d}}-1\right)
\end{array}
$$

where $\overline{N_{d}}=N_{d}-\alpha$ with a parameter $\alpha$. Our $V^{D C}$ formula can be derived from $E^{D C}\left(V^{D C}=\partial E^{D C} / \partial N_{d}\right)$ and allow site-dependent potentials similarly as the FLL form. The conventional FLL DC form is recovered by setting $\alpha=0$. By increasing $\alpha, V^{D C}$ can be close to the nominal $V^{D C}$ value as $\overline{N_{d}}$ approaches to $N_{d}^{0}\left(\alpha=N_{d}-\right.$
$\left.N_{d}^{0}\right)$.

Another modified form of $V^{D C}$ for DFT+DMFT was also suggested as below since the $U$ value used in the FLL form can be smaller than the Hubbard $U\left(U^{\prime}<U\right)$ to allow the smaller $V^{D C}$ potential than the FLL one:

$$
\begin{array}{r}
E^{D C}=\frac{U^{\prime}}{2} \cdot N_{d} \cdot\left(N_{d}-1\right)-\frac{J}{4} \cdot N_{d} \cdot\left(N_{d}-2\right) \\
V^{D C}=U^{\prime} \cdot\left(N_{d}-\frac{1}{2}\right)-\frac{J}{2} \cdot\left(N_{d}-1\right)
\end{array}
$$

where $U^{\prime}=U-\alpha$ with a parameter $\alpha$. Here, the role of $\alpha$ is the same as the one in Eq.16. Namely, the covalency effect can be reduced by increasing the $\alpha$ value. It has been shown that using $U^{\prime}=U-0.2 \mathrm{eV}$ can successfully reproduce the structural and electronic phase diagram of rare-earth nickelates $\stackrel{42,49}{ }$.
1 M. Imada, A. Fujimori, and Y. Tokura, Rev. Mod. Phys. 70, 1039 (1998)

2 N. B. Ivanova, S. G. Ovchinnikov, M. M. Korshunov, I. M. Eremin, and N. V. Kazak, Physics-Uspekhi 52, 789 (2009)

3 T. Saitoh, T. Mizokawa, A. Fujimori, M. Abbate, Y. Takeda, and M. Takano, Phys. Rev. B 55, 4257 (1997)

4 M. Abbate, J. C. Fuggle, A. Fujimori, L. H. Tjeng, C. T. Chen, R. Potze, G. A. Sawatzky, H. Eisaki, and S. Uchida, Phys. Rev. B 47, 16124 (1993)

5 R. F. Klie, J. C. Zheng, Y. Zhu, M. Varela, J. Wu, and C. Leighton, Phys. Rev. Lett. 99, 047203 (2007)

${ }^{6}$ C. Zobel, M. Kriener, D. Bruns, J. Baier, M. Grüninger, T. Lorenz, P. Reutler, and A. Revcolevschi, Phys. Rev. B 66, 020402(R) (2002).

7 M. W. Haverkort, Z. Hu, J. C. Cezar, T. Burnus, H. Hartmann, M. Reuther, C. Zobel, T. Lorenz, A. Tanaka, N. B. Brookes, H. H. Hsieh, H.-J. Lin, C. T. Chen, and L. H. Tjeng, Phys. Rev. Lett. 97, 176405 (2006).

8 A. Podlesnyak, S. Streule, J. Mesot, M. Medarde, E. Pomjakushina, K. Conder, A. Tanaka, M. W. Haverkort, and D. I. Khomskii, Phys. Rev. Lett. 97, 247208 (2006)

9 P. M. Raccah and J. B. Goodenough, Phys. Rev. 155, 932 (1967).

10 R. A. Bari and J. Sivardière, Phys. Rev. B 5, 4466 (1972)

11 M. Sears-Rodrguez and J. Goodenough, Journal of Solid State Chemistry 116, 224 (1995).

12 T. Kyômen, Y. Asaka, and M. Itoh, Phys. Rev. B 71, 024418 (2005).

13 A. A. Yaroslavtsev, M. Izquierdo, R. Carley, M. E. Dávila, A. A. Ünal, F. Kronast, A. Lichtenstein, A. Scherz, and S. L. Molodtsov, Phys. Rev. B 93, 155137 (2016).

14 K. Asai, O. Yokokura, N. Nishimori, H. Chou, J. M. Tranquada, G. Shirane, S. Higuchi, Y. Okajima, and K. Kohn, Phys. Rev. B 50, 3025 (1994)

15 K. Berggold, M. Kriener, P. Becker, M. Benomar, M. Reuther, C. Zobel, and T. Lorenz, Phys. Rev. B 78, 134402 (2008).

16 Knízek, K., Jirák, Z., Hejtmánek, J., Veverka, M., Marysko, M., Maris, G., and Palstra, T. T.M., Eur. Phys. J. B 47, 213 (2005).
$17 \mathrm{P}$. Phys. Rev. B 66, 094408 (2002)

18 J.-Q. Yan, J.-S. Zhou, and J. B. Goodenough, Phys. Rev. B 69, 134409 (2004).

19 V. Gnezdilov, V. Fomin, A. V. Yeremenko, K.-Y. Choi, Y. Pashkevich, P. Lemmens, S. Shiryaev, G. Bychkov, and S. Barilo, Low Temperature Physics 32, 162 (2006).

20 D. Fuchs, C. Pinta, T. Schwarz, P. Schweiss, P. Nagel, S. Schuppler, R. Schneider, M. Merz, G. Roth, and H. v. Löhneysen, Phys. Rev. B 75, 144402 (2007).

21 J. W. Freeland, J. X. Ma, and J. Shi, Applied Physics Letters 93, 212501 (2008).

22 W. S. Choi, J.-H. Kwon, H. Jeen, J. E. HamannBorrero, A. Radi, S. Macke, R. Sutarto, F. He, G. A. Sawatzky, V. Hinkov, M. Kim, and H. N. Lee, Nano Letters 12, 4966 (2012)

23 G. E. Sterbinsky, R. Nanguneri, J. X. Ma, J. Shi, E. Karapetrova, J. C. Woicik, H. Park, J.-W. Kim, and P. J. Ryan, Phys. Rev. Lett. 120, 197201 (2018)

24 J. Fujioka, Y. Yamasaki, H. Nakao, R. Kumai, Y. Murakami, M. Nakamura, M. Kawasaki, and Y. Tokura, Phys. Rev. Lett. 111, 027206 (2013)

25 M. A. Korotin, S. Y. Ezhov, I. V. Solovyev, V. I. Anisimov, D. I. Khomskii, and G. A. Sawatzky, Phys. Rev. B 54, 5309 (1996)

26 K. Knížek, P. Novák, and Z. Jirák, Phys. Rev. B 71, 054420 (2005).

27 K. Knížek, Z. Jirák, J. Hejtmánek, and P. Novák, Journal of Physics: Condensed Matter 18, 3285 (2006).

28 K. Knížek, Z. Jirák, J. Hejtmánek, P. Novák, and W. Ku, Phys. Rev. B 79, 014430 (2009).

29 A. Georges, G. Kotliar, W. Krauth, and M. J. Rozenberg, Rev. Mod. Phys. 68, 13 (1996)

30 G. Kotliar, S. Y. Savrasov, K. Haule, V. S. Oudovenko, O. Parcollet, and C. A. Marianetti, Rev. Mod. Phys. 78, 865 (2006).

31 L. Craco and E. Müller-Hartmann, Phys. Rev. B 77, 045130 (2008).

32 G. Zhang, E. Gorelov, E. Koch, and E. Pavarini, Phys. Rev. B 86, 184413 (2012).

33 V. Křápek, P. Novák, J. Kuneš, D. Novoselov, 
D. M. Korotin, and V. I. Anisimov, Phys. Rev. B 86, 195104 (2012).

34 B. Chakrabarti, T. Birol, Phys. Rev. Materials 1, 064403 (2017)

35 J. Kuneš and V. and K. Haule, Phys. Rev. Lett. 106, 256401 (2011)

36 M. Karolak, M. Izquierdo, S. L. Molodtsov, and A. I. Lichtenstein, Phys. Rev. Lett. 115, 046401 (2015)

37 G. Kresse and J. Hafner, Phys. Rev. B 47, 558 (1993).

38 G. Kresse and J. Hafner, Phys. Rev. B 49, 14251 (1994).

39 G. Kresse and J. Furthmuller, Comput. Mat. Sci. 6, 15 (1996).

40 G. Kresse and J. Furthmuller, Phys. Rev. B 54, 11169 (1996).

41 J. P. Perdew, K. Burke, and M. Ernzerhof, Phys. Rev. Lett. 77, 3865 (1996).

42 H. Park, A. J. Millis, and C. A. Marianetti, Phys. Rev. B 90, 235103 (2014).

43 V. Singh, U. Herath, B. Wah, X. Liao, A. H. Romero, and H. Park, arXiv:2002.00068 (2020).

44 A. A. Mostofi, J. R. Yates, G. Pizzi, Y.-S. Lee, I. Souza, D. Vanderbilt, and N. Marzari, Computer Physics Communications 185, 2309 (2014).
45 N. Marzari, A. A. Mostofi, J. R. Yates, I. Souza, and D. Vanderbilt, Rev. Mod. Phys. 84, 1419 (2012)

46 K. Haule, Phys. Rev. B 75, 155113 (2007)

47 E. Gull, A. J. Millis, A. I. Lichtenstein, A. N. Rubtsov, M. Troyer, and P. Werner, Rev. Mod. Phys. 83, 349 (2011).

48 V. I. Anisimov, J. Zaanen, and O. K. Andersen, Phys. Rev. B 44, 943 (1991)

49 H. Park, A. J. Millis, and C. A. Marianetti, Phys. Rev. B 89, 245133 (2014).

50 K. Haule, T. Birol, and G. Kotliar, Phys. Rev. B 90, 075136 (2014).

51 X. Wang, M. J. Han, L. de' Medici, H. Park, C. A. Marianetti, and A. J. Millis, Phys. Rev. B 86, 195136 (2012)

${ }^{52}$ H. T. Dang, A. J. Millis, and C. A. Marianetti, Phys. Rev. B 89, 161113(R) (2014)

53 K. Haule, Phys. Rev. Lett. 115, 196403 (2015)

${ }^{54}$ H. Park, A. J. Millis, and C. A. Marianetti, Phys. Rev. B 92, 035146 (2015).

55 H. Park, A. J. Millis, and C. A. Marianetti, Phys. Rev. Lett. 109, 156402 (2012)

56 A. Chainani, M. Mathew, and D. D. Sarma, Phys. Rev. B 46, 9976 (1992) 